\title{
Retirada do fósforo suplementar na estação seca para vacas Nelore em pastejo
}

\author{
Ériklis Nogueira(1) Maria Luiza Franceschi Nicodemo(2), Luiz Roberto Lopes de S' Thiago(3), \\ Sheila da Silva Moraes ${ }^{(3)}$, Manuel Cláudio Motta Macedo ${ }^{(3)}$ e Jairo Mendes Vieira ${ }^{(3)}$
}

\begin{abstract}
(1)Embrapa Pantanal, Caixa Postal 109, CEP 79320-900 Corumbá, MS. E-mail: eriklis@cpap.embrapa.br (2)Embrapa Pecuária Sudeste, Caixa Postal 339, CEP 13560-970 São Carlos, SP. E-mail: mlnicodemo@cppse.embrapa.br (3)Embrapa Gado de Corte, Caixa Postal 154, CEP 79002-970, Campo Grande, MS. E-mail: thiago@cnpgc.embrapa.br, moraes_sheila@yahoo.com.br, macedo@cnpgc.embrapa.br, jairomvieira@bol.com.br
\end{abstract}

Resumo - O objetivo deste trabalho foi avaliar o desempenho reprodutivo de vacas Nelore em pastos de capim-marandu, após a retirada do fosfato bicálcico da mistura mineral, na estação seca. Sessenta matrizes receberam, durante seis anos, mistura mineral completa na estação chuvosa. Na estação seca, os tratamentos consistiram de: mistura mineral completa (MMC); MMC sem fosfato bicálcico (MM); e MM + concentrado. As pastagens foram manejadas de forma a não limitar a disponibilidade de matéria seca. A retirada do fosfato bicálcico do suplemento mineral, durante a estação seca, não prejudicou o desempenho reprodutivo de vacas, avaliado pela taxa de prenhez, intervalo de partos e retorno da atividade cíclica ovariana. Vacas que receberam concentrado na estação seca pariram em melhor condição corporal; vacas primíparas arraçoadas apresentaram menor número de dias vazios do que as vacas primíparas dos demais tratamentos. A retirada do fosfato bicálcico suplementar, fonte de fósforo e cálcio, na estação seca, não prejudica o desempenho de vacas multíparas em pastejo de capim-marandu.

Termos para indexação: Brachiaria brizantha, atividade ovariana, reprodução, taxa de prenhez.

\section{Phosphate supplementation withdrawal during dry season for Nelore range cows}

\begin{abstract}
The objective of this work was to evaluate the reproductive performance of Nelore cows at 'Marandu' palisade grass paddocks, after dicalcium phosphate withdrawal from the mineral supplement in the dry season. Sixty breeding cows received, during six years, a complete mineral mixture in the wet season. In the dry season, the treatments consisted of: a complete mineral mixture (MMC); MMC without dicalcium phosphate (MM); and MM plus a concentrate. Pastures were managed in order to allow for adequate dry matter availability. The removal of dicalcium phosphate from the mineral supplement, during the dry season, did not affect the animal reproduction performance which was evaluated as pregnancy rates, delivery intervals and return of oestrous cycles. Cows receiving concentrate in the dry season had higher body score at delivery; primiparous cows which fed concentrate showed a lower number of days open, in comparison to the primiparous ones of the other treatments. Dicalcium phosphate withdrawal from the mineral supplement, source of phosphorus and calcium, during the dry season, does not affect the reproductive performance of multiparous range cows grazing 'Marandu' palisade grass.
\end{abstract}

Index terms: Brachiaria brizantha, oestrous cycle, reproduction, pregnancy rate.

\section{Introdução}

Bovinos em pastos de braquiária, na região do Cerrado, geralmente precisam de fósforo (P) suplementar na dieta, porque o capim não é capaz de fornecê-lo suficientemente para atender às exigências nutricionais desses animais. A deficiência de $\mathrm{P}$ foi associada à queda do ganho de peso e da produção de leite, bem como à redução do desempenho reprodutivo (Karn, 2001; Ferris, 2010). É possível elevar o P da dieta de bovinos em pastejo via fertilização da forrageira ou pela suplementação direta. Em áreas extensas, a suplementação direta é considerada economicamente mais viável (McIvor et al., 2011). A fonte de P pode corresponder a quase metade do custo de produção do suplemento mineral. A procura por redução de custos levou à avaliação de fosfatos de rocha ou de uso agrícola na suplementação animal. Outra possibilidade está na redução da oferta de $\mathrm{P}$, em situações específicas, para vacas em reprodução (Knowlton et al., 2004; Lopez et al., 2004a). 
$\mathrm{Na}$ estação seca, a braquiária predominante nas regiões de solo de baixa fertilidade do Brasil Central apresenta concentrações típicas de P de 0,09 a $0,11 \%$ de matéria seca (Moraes, 2001). Na deficiência de $\mathrm{P}$, o organismo usa mecanismos adaptativos para manter as concentrações de P nos tecidos adequadas, por sua vez, para manter suas funções e pode mobilizar as reservas ósseas (Karn, 2001; Satter et al., 2005). Se essa situação prevalecer por muito tempo, os processos fisiológicos são prejudicados.

Assim, postulou-se que as vacas não seriam prejudicadas com a retirada do $\mathrm{P}$ e $\mathrm{Ca}$ suplementar da mistura mineral apenas na estação seca, ao se considerarem: a coincidência da fase de menores exigências de $\mathrm{P}$ pelas vacas na estação seca, para fêmeas que parem no início da estação chuvosa (National Research Council, 1996; Karn, 2001); a queda na qualidade e massa de capim na estação seca, que limita a disponibilidade de proteína e energia; e a possibilidade de mobilização de $\mathrm{P}$ do osso (Karn, 2001; Knowlton et al., 2004; Ekelund et al., 2006). Considerou-se, também, que a correção das deficiências de energia e de proteína, durante a seca, poderia tornar a suplementação de $\mathrm{P}$ mais eficiente.

O objetivo deste trabalho foi avaliar o desempenho reprodutivo de vacas Nelore em pastos de capimmarandu, em área de cerrado, em resposta à retirada do fosfato bicálcico da mistura mineral na estação seca.

\section{Material e Métodos}

O experimento foi realizado na Embrapa Gado de Corte, Campo Grande, MS, a $20^{\circ} 27^{\prime} \mathrm{S}, 54^{\circ} 37^{\prime} \mathrm{W}$ e à altitude de $530 \mathrm{~m}$. O clima, segundo a classificação de Köppen, é do tipo Aw, tropical úmido, com estação chuvosa no verão e seca no inverno. A precipitação média anual é de $1.500 \mathrm{~mm}$, e os meses de menor precipitação são junho, julho e agosto. A temperatura média anual oscila de 19 a $25^{\circ} \mathrm{C}$.

Sessenta novilhas Nelore com diagnóstico positivo de prenhez, idade média de 42 meses, peso de $379 \mathrm{~kg}$ e escore corporal (1-9) de 5,3, foram distribuídas em três tratamentos, em blocos de dez vacas, de acordo com o ano de nascimento (1994 e 1995), no total de 20 fêmeas por tratamento. Os períodos correspondentes ao primeiro ano experimental abrangeram a gestação em 1998, o parto em 1998 e a lactação em 1999; o segundo ano experimental abrangeu a gestação em
1999, o parto em 1999 e a lactação em 2000, e assim por diante. O sexto e último período experimental abrangeu a gestação em 2003, o parto em 2003 e a lactação em 2004. O experimento foi implantado em três piquetes de 22 ha com 'Marandu' (Urochloa brizantha, Syn. Brachiaria brizanta), formados há cerca de dez anos, em Latossolo Vermelho-Escuro plano, imperfeitamente drenado. Os piquetes foram vedados em fevereiro de 1998, e as fêmeas foram introduzidas em julho de 1998. O experimento durou de 18/8/1998 a 28/5/2004 (68 meses). A área foi submetida à gessagem e calagem (613 $\mathrm{kg} \mathrm{ha}^{-1}$ de gesso agrícola, e 2,27 $\mathrm{Mg} \mathrm{ha}^{-1}$ de calcário dolomítico), em setembro de 1998, e à adubação (500 kg ha-1 de NPK 2-50-20; $110 \mathrm{~kg} \mathrm{ha}^{-1}$ de ureia no total de $59,5 \mathrm{~kg} \mathrm{~N} ; 250 \mathrm{~kg} \mathrm{P}_{2} \mathrm{O}_{5}$ e $100 \mathrm{~kg} \mathrm{~K}_{2} \mathrm{O}$ ) em janeiro de 1999. Foi feita nova calagem (calcário dolomítico $80 \%$ PRNT, 1,3 $\mathrm{Mg} \mathrm{ha}^{-1}$ ), em outubro de 2001, e adubação (350 $\mathrm{kg} \mathrm{ha}^{-1}$ de NPK 20-10-20, no total de $70 \mathrm{~kg} \mathrm{~N}, 35 \mathrm{~kg} \mathrm{P}_{2} \mathrm{O}_{5}$ e $70 \mathrm{~kg} \mathrm{~K}_{2} \mathrm{O}$ ) em dezembro de 2001. Os piquetes dispunham de água à vontade, fornecida em pilhetas e cochos cobertos para mistura mineral. Os lotes de animais foram rotacionados nos piquetes a cada 15 dias.

A estação de reprodução (EM) com monta natural durou 90 dias - de janeiro a março -, com concentração dos partos no início da estação chuvosa. Utilizaram-se três touros, distribuídos nos tratamentos após a avaliação andrológica, rotacionados quinzenalmente entre os lotes. Na estação de monta das vacas primíparas, foi realizada a desmama interrompida, de 48 a 72 horas, para estimular o retorno ao cio. O diagnóstico de gestação foi realizado em maio/junho, por meio de palpação retal. Os bezerros foram desmamados entre seis e sete meses de idade. No intervalo entre os partos, consideraram-se apenas os dados de vacas que pariram em anos consecutivos.

Os tratamentos foram: MMC, mistura mineral completa no ano todo $(57,923 \%$ de fosfato bicálcico; $2,041 \%$ de sulfato de zinco; $0,454 \%$ de sulfato de cobre; $0,019 \%$ de sulfato de cobalto; $0,008 \%$ de iodato de potássio; $0,005 \%$ de selenito de sódio; $7,24 \%$ de flor de enxofre; $31,31 \%$ de cloreto de sódio; e $1 \%$ de melaço em pó); MM, na estação das águas foi fornecido $\mathrm{MMC}$ e, na estação seca, a mistura mineral (MMC) sem fosfato bicálcico; e MMR, na estação das águas foi fornecido $\mathrm{MMC}$ e, na estação seca, a mistura mineral (MMC) sem fosfato bicálcico + suplemento proteico-energético ( $800 \mathrm{~g}$ de farelo de soja; $1.500 \mathrm{~g}$ de 
grão de milho triturado e $32 \mathrm{~g}$ de carbonato de cálcio; o fornecimento correspondeu a $0,55 \%$ do peso-vivo). A oferta de 2,3 $\mathrm{kg}$ de ração por vaca buscou atender às necessidades de proteína e energia; a ração foi totalmente consumida. O controle de consumo da mistura mineral foi mensal, obtido pela diferença de pesagem entre o fornecido e a sobra. Os períodos de seca compreenderam: $18 / 8$ a 14/10/1998, 9/6 a $3 / 11 / 1999,13 / 6$ a 11/9/2000, 27/6 a 28/9/2001, 12/6 a 22/10/2002 e 24/6 a 22/9/2003.

Quanto ao desempenho animal, foram avaliados: o peso das matrizes ao parto e à desmama; a condição corporal ao parto e à desmama, em escala 1 a 9 de Machado et al. (2008); e o peso dos bezerros ao nascer e à desmama. Foram feitas coletas aleatórias de sangue, de sete a dez vacas por tratamento; o sangue foi colhido via jugular, em frascos heparinizados, e foi centrifugado a $3.000 \mathrm{rpm}$ por $15 \mathrm{~min}$; em seguida, foi feita a separação do plasma e o armazenamento em alíquotas a $-20^{\circ} \mathrm{C}$ até a análise de fósforo inorgânico (Fick et al., 1980). O intervalo parto-concepção e a taxa de gestação, ao final da EM, foi calculado. $\mathrm{O}$ aprofundamento das investigações de aspectos reprodutivos, com acompanhamento do puerpério por meio de ultrassonografia do trato genital, iniciou-se no quinto ano experimental, aos 30 dias após o parto (dezembro de 2002), e foram repetidas semanalmente até a observação de ciclicidade, por meio da avaliação da progesterona plasmática e da observação de corpo lúteo $(\mathrm{CL})$, por ultrassonografia transretal, finalizadas em fevereiro de 2003. As coletas de sangue foram realizadas por punção da veia jugular, em tubos com EDTA, no período da manhã. Os tubos foram mantidos em banho de gelo até a separação do plasma, armazenado a $-18^{\circ} \mathrm{C}$. As análises de progesterona (P4) foram realizadas por radioimunoensaio, com kits coat-a-count (DPC- Medlab, Los Angeles, CA, EUA) na Universidade Estadual Paulista, Botucatu, SP. Glicose, albumina e ureia no plasma também foram analisadas com a utilização de kits comerciais Lab Test (Lab Test, Belo Horizonte, MG, Brasil) que incluíram reações enzimáticas colorimétricas com o uso de espectrofotômetro. Foram consideradas cíclicas as vacas que apresentaram $\mathrm{CL}$ visível à ultrassonografia e com níveis plasmáticos de progesterona acima de $1 \mathrm{ng} \mathrm{mL} \mathrm{mL}^{-1}$.

A determinação da qualidade da forragem foi feita no final da estação seca e das águas, em amostras obtidas por simulação de pastejo de aproximadamente $500 \mathrm{~g}$, coletadas após a observação do hábito de pastejo dos animais. Foram realizadas análises de proteína bruta e digestibilidade in vitro da matéria orgânica (Harris, 1970) e Ca e P (Fick et al., 1980). Amostras para avaliação da disponibilidade de matéria seca (MS) foram colhidas conforme Haydock \& Shaw (1975). A taxa de lotação foi calculada pela divisão do somatório do peso dos animais, em cada piquete, pela respectiva área do piquete. Os valores foram expressos em unidade animal (UA) por hectare, de modo que uma UA correspondeu a $450 \mathrm{~kg}$ de peso corporal. A oferta de forragem foi calculada pela divisão da massa de forragem $\left(\mathrm{kg} \mathrm{ha}^{-1}\right)$ pelo peso do animal $\left(\mathrm{kg} \mathrm{ha}^{-1}\right)$.

Utilizou-se o delineamento de blocos ao acaso, com parcelas subdivididas no tempo em que os blocos consistiram do ano de nascimento das vacas (1994 e 1995). Os tratamentos foram alocados na parcela principal, as épocas de amostragem na subparcela, e o ano experimental à subsubparcela. Não foram estabelecidas repetições para os pastos. $\mathrm{O}$ modelo estatístico utilizado foi $\mathrm{Y}_{\mathrm{ijk}}=\mu+\mathrm{A}_{\mathrm{i}}+\mathrm{P}_{\mathrm{j}}+\mathrm{T}_{\mathrm{k}}+\varepsilon_{\mathrm{ijk}}$, em que: $\mu$ é a média dos tratamentos; $A_{i}$ é o efeito do ano de nascimento das vacas $\mathrm{i} ; \mathrm{P}_{\mathrm{j}}$ é o efeito das épocas de amostragem j; $\mathrm{T}_{\mathrm{k}}$ é o efeito do ano de amostragem $\mathrm{k}$; e $\varepsilon_{\mathrm{ijk}}$ é o erro aleatório.

As análises de variância foram realizadas com o procedimento GLM do SAS. Quando houve diferença significativa pelo teste $\mathrm{F}$, as médias foram comparadas pelo teste de Tukey-Kramer, a 5\% de probabilidade, e quando a significância entre fontes de variação esteve entre 5 e $9 \%$, desdobraram-se os graus de liberdade em contrastes ortogonais pré-definidos, tais como: MMC x MM; MMC e MM x MMR. Na análise de variância, foram estudadas as interações entre bloco e tratamento e entre tratamento e ano experimental. Para os dados de taxa de gestação, foi realizada a análise pelo qui-quadrado. Para glicose, ureia, fósforo inorgânico, albumina e progesterona plasmática, as médias foram comparadas pelo teste de SNK, a 5\% de probabilidade.

\section{Resultados e Discussão}

As médias de peso corporal, entre os tratamentos (Tabela 1), não diferiram $(\mathrm{p}>0,05)$, bem como o peso do bezerro ao nascer ( $p>0,05)$. Também não se observaram diferenças entre as médias de peso das vacas à desmama ( $>0,05)$, bem como nos pesos dos bezerros à desmama $(p>0,05)$. As vacas arraçoadas 
apresentaram melhor condição corporal no parto do que aquelas dos demais tratamentos $(p<0,05)$. Pimentel et al. (2011) observaram maior consumo de capim-marandu em vacas que receberam $2,0 \mathrm{~kg}$ de suplemento, na estação seca, em comparação àquelas que receberam apenas sal mineral. Isso foi atribuído ao efeito associativo positivo da suplementação no consumo do pasto. $\mathrm{O}$ arraçoamento pode contribuir para o aproveitamento efetivo da forragem disponível e contribuir para o melhor desempenho.
As vacas estavam em pastagens com massa de matéria seca adequada capaz de lhes permitir a seleção do material de melhor qualidade, especialmente na estação chuvosa (Tabela 2). A capacidade de suporte, estimada na região para o capim- marandu, variou de 1,33 a 2,07 UA ha ${ }^{-1}$. Embora a taxa de lotação possa variar entre locais, com os níveis de fertilização, e entre períodos do ano e ao longo do tempo (Euclides \& Euclides Filho, 1998), a taxa de lotação média utilizada no presente trabalho $\left(0,94 \mathrm{UA} \mathrm{ha}^{-1}\right)$ foi

Tabela 1. Peso corporal de vacas ao parto $(\mathrm{PCp})$ e à desmama ( $\mathrm{PCd})$, condição corporal das vacas ao parto $(\mathrm{CCp})$ e à desmama $(\mathrm{CCd})$, peso dos bezerros ao nascer $(\mathrm{PNb})$ e à desmama $(\mathrm{PDb})$, e contrastes entre os efeitos dos tratamentos e essas variáveis e valores $\mathrm{p}$ associados ${ }^{(1)}$.

\begin{tabular}{|c|c|c|c|c|c|c|}
\hline \multirow[t]{2}{*}{ Tratamento } & PCp & $\mathrm{PCd}$ & $\mathrm{CCp}$ & $\mathrm{CCd}$ & $\mathrm{PNb}$ & $\mathrm{PDb}$ \\
\hline & \multicolumn{2}{|c|}{----------- $(\mathrm{kg}$ ) --------------- } & \multicolumn{2}{|c|}{------------- (1-9) ------------- } & \multicolumn{2}{|c|}{ 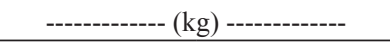 } \\
\hline $\mathrm{MMC}$ & $399,98 \pm 4,12 \mathrm{a}$ & $414,37 \pm 5,15 \mathrm{a}$ & $5,16 \pm 0,56 b$ & $5,22 \pm 0,07 \mathrm{a}$ & $29,26 \pm 0,30 \mathrm{a}$ & $180,6 \pm 2,05 \mathrm{a}$ \\
\hline MM & $418,32 \pm 4,30 \mathrm{a}$ & $429,15 \pm 5,01 \mathrm{a}$ & $5,15 \pm 0,63 b$ & $5,17 \pm 0,07 \mathrm{a}$ & $29,66 \pm 0,30 \mathrm{a}$ & $184,6 \pm 2,16 \mathrm{a}$ \\
\hline MMR & $437,97 \pm 4,31 \mathrm{a}$ & $439,72 \pm 5,10 \mathrm{a}$ & $5,60 \pm 0,64 \mathrm{a}$ & $5,52 \pm 0,07 \mathrm{a}$ & $29,21 \pm 0,31 \mathrm{a}$ & $179,8 \pm 2,14 \mathrm{a}$ \\
\hline Contrastes $^{(2)}$ & - & - & - & Valor $\mathrm{p}^{(3)}$ & - & - \\
\hline MMC x. MM & 0,3368 & 0,2359 & 0,9621 & 0,6742 & 0,3664 & 0,1069 \\
\hline MMC, MM x. MMR & 0,1546 & 0,1424 & $0,0354 *$ & 0,0639 & 0,5016 & 0,1503 \\
\hline
\end{tabular}

${ }^{(1)}$ Médias \pm desvio-padrão seguidas de letras iguais, nas colunas, não diferem entre si, pelo teste de Tukey-Kramer, a 5\% de probabilidade. MMC, mistura mineral completa; MM, MMC sem fosfato bicálcico; MMR, MM + concentrado. ${ }^{(2)}$ Contrastes entre os efeitos dos tratamentos. ${ }^{(3)}$ Valor $\mathrm{p}$, probabilidade de erro tipo I, associada ao teste F para o contraste.

Tabela 2. Estimativa de massa de matéria seca (MS) e concentrações de $\mathrm{P}, \mathrm{Ca}$, proteína bruta (PB) na MS e digestibilidade in vitro da matéria orgânica (DIVMO) do pasto.

\begin{tabular}{|c|c|c|c|c|c|c|c|}
\hline Ano & Data de suplementação & Data de coleta & $\begin{array}{c}\text { MS } \\
\left(\mathrm{kg} \mathrm{ha}^{-1}\right)\end{array}$ & $\begin{array}{c}\mathrm{P} \\
-\end{array}$ & PB & $\mathrm{Ca}$ & DIVMO \\
\hline \multirow{3}{*}{1} & \multirow{3}{*}{$11 / 8$ a $14 / 10 / 1998$} & Out./1998 & 5.477 & 0,14 & 9,60 & 0,63 & 57,00 \\
\hline & & Fev./1999 & 5.693 & 0,18 & 11,20 & 0,37 & 54,60 \\
\hline & & Jun./1999 & 7.364 & 0,10 & 5,30 & 0,56 & 44,20 \\
\hline \multirow{3}{*}{2} & \multirow{3}{*}{ 9/6 a 2/11/1999 } & Out./1999 & 3.642 & 0,19 & 12,50 & 0,28 & 70,30 \\
\hline & & Fev./2000 & 3.360 & 0,14 & 9,30 & 0,34 & 61,60 \\
\hline & & Jun./2000 & 3.727 & 0,16 & 7,00 & 0,57 & 47,10 \\
\hline \multirow{3}{*}{3} & \multirow{3}{*}{$13 / 6$ a $11 / 9 / 2000$} & Set./2000 & 3.056 & 0,17 & 11,20 & 0,51 & 64,00 \\
\hline & & Dez./2000 & 3.619 & 0,10 & 8,30 & 0,40 & 57,80 \\
\hline & & Jun./2001 & 2.771 & 0,17 & 10,40 & 0,46 & 56,50 \\
\hline \multirow{3}{*}{4} & \multirow{3}{*}{$11 / 6$ a 9/10/2001 } & Out./2001 & 1.956 & 0,14 & 10,40 & 0,51 & 64,70 \\
\hline & & Fev./2001 & 3.296 & 0,15 & 10,90 & 0,40 & 61,60 \\
\hline & & Jun./2002 & 5.678 & 0,07 & 5,70 & 0,66 & 41,70 \\
\hline \multirow{3}{*}{5} & \multirow{3}{*}{$21 / 6$ a $22 / 10 / 2002$} & Out./2002 & 4.102 & 0,15 & 11,50 & 0,32 & 69,90 \\
\hline & & Fev./2003 & 3.355 & 0,16 & 8,00 & 0,44 & 49,60 \\
\hline & & Jun./2003 & 3.071 & 0,15 & 8,80 & 0,50 & 60,60 \\
\hline \multirow{3}{*}{6} & \multirow{3}{*}{$24 / 6$ a $22 / 9 / 2003$} & Set./2003 & 3.563 & 0,13 & 9,20 & 0,36 & 63,90 \\
\hline & & Fev./2004 & 3.213 & 0,16 & 7,80 & 0,38 & 55,80 \\
\hline & & Jun./2004 & 3.753 & $-(1)$ & - & - & - \\
\hline Média & & & 3.928 & 0,14 & 9,20 & 0,45 & 57,70 \\
\hline
\end{tabular}

${ }^{(1)}$ Dado não disponível. 
relativamente leve; a oferta de forragem média foi de 9,28 $\mathrm{kg}$ de $\mathrm{MS} \mathrm{kg}^{-1}$ de peso corporal, o que indica abundância de massa disponível. Santos et al. (2009) relataram valores de oferta de braquiária em pastagens diferidas, que variaram de 3,46 a 7,65 kg de $\mathrm{MS} \mathrm{kg}^{-1}$ de peso corporal. Os valores de oferta elevados foram considerados necessários, para evitar a redução no desempenho animal, em razão do baixo valor nutritivo da forragem na estação seca. Em meados de 2001, os animais terminaram a estação seca com baixa disponibilidade de massa de capim, que foi corrigida com calagem e adubação de cobertura.

$\mathrm{O}$ valor nutritivo da forragem pode ser avaliado pela sua digestibilidade e pelos seus teores de proteína bruta, estreitamente relacionados com o consumo de matéria seca. $\mathrm{O}$ pasto apresentou variações nas concentrações de proteína bruta e de matéria orgânica digestível compatíveis com aquelas geralmente observadas em pastagens tropicais (Tabela 2). Em junho, dois atributos da forragem podem ter prejudicado o consumo de matéria seca: os teores de proteína bruta inferiores a 7\% e os elevados conteúdos de parede celular na matéria seca (Alves et al., 2001). A concentração de cálcio na forrageira era adequada à nutrição das vacas, com teores acima de $0,28 \%$, sem necessitar de correção via suplemento (National Research Council, 1996).

As vacas obtiveram $P$ para atender às suas demandas por meio do consumo de pasto e do suplemento mineral e do consumo de ração em MMR. Para efeito de cálculo, considerou-se o consumo de MS variável, correspondente a $2 \%$ do peso vivo para "vacas secas" e a $2,3 \%$ do peso vivo para vacas em lactação. O consumo médio de suplemento mineral (UA por dia) foi de $64 \mathrm{~g}$ na estação chuvosa; na estação seca, as médias ponderadas de consumo foram de $50 \mathrm{~g}$ (MMC), $38 \mathrm{~g}(\mathrm{MM})$ e $24 \mathrm{~g}$ (MMR). A ingestão aproximada de $\mathrm{P}$ (g por dia), nos diferentes tratamentos durante o ciclo reprodutivo foi calculada e comparada às exigências nutricionais estimadas de $\mathrm{P}$ pelo National Research Council (1996) (Tabela 3). Apenas as vacas do tratamento $\mathrm{MM}$ não atenderam às demandas do terço final da gestação e apresentaram consumo marginal de P no terço médio da gestação.

Há relatos de aumento da reabsorção de osso (Ekelund et al., 2006) e de redução da formação de osso (Puggaard et al., 2011) com o aumento da demanda por $\mathrm{P}$ em vacas, por mecanismos pouco conhecidos (Karn, 2001). Assim, as vacas poderiam utilizar as reservas ósseas para manter a integridade dos processos metabólicos, em períodos de deficiência de $\mathrm{P}$, como no tratamento MM durante a estação seca, desde que recebessem dieta adequada para reabilitação de suas reservas em outra fase do ciclo (Ferris et al., 2010). Tal fato pode ser evidenciado pelos níveis plasmáticos de $\mathrm{P}$ (Tabela 4), que estavam dentro dos limites fisiológicos para bovinos. Os valores de referência para P inorgânico circulante, para bovinos de corte, variam de 4,3 a 7,7 $\mathrm{mg} \mathrm{dL}^{-1}$; o valor crítico é de $3 \mathrm{mg} \mathrm{dL}^{-1}$ (Diaz González et al., 2000). O arraçoamento permitiu às vacas aumentar as reservas corporais de $\mathrm{P}(\mathrm{p}<0,05)$.

A deficiência severa de $\mathrm{P}$ é associada a desordens reprodutivas em bovinos, mas a infertilidade geralmente ocorre depois de outros sintomas clínicos. Manifestações clássicas da deficiência de $\mathrm{P}$ envolvem alterações no estro, com baixa expressão dos sinais desse (Nicodemo et al., 2008). Grande parte dos estudos recentes, relacionados à suplementação de fósforo, é sobre vacas leiteiras de alta produção. Os estudos mostraram bom desempenho reprodutivo com concentrações de $\mathrm{P}$, que atendiam marginalmente às exigências nutricionais desses animais ( $\mathrm{Wu} \mathrm{\&}$ Satter, 2000; Lopez et al., 2004b; Tallam et al., 2005; Ferris et al., 2010). Os teores mais baixos de P, nas dietas estudadas, foram considerados adequados pelos

Tabela 3. Estimativas das demandas e consumos de P nas dietas de vacas em reprodução.

\begin{tabular}{|c|c|c|c|c|c|c|c|c|c|c|}
\hline \multirow{2}{*}{$\begin{array}{l}\text { Meses após } \\
\text { o parto }\end{array}$} & \multirow[t]{2}{*}{ Estádio fisiológico } & \multirow[t]{2}{*}{ Período } & \multirow{2}{*}{$\begin{array}{c}\text { Demanda } \\
\text { de } \mathrm{P}^{(1)}\end{array}$} & \multirow{2}{*}{$\begin{array}{c}\text { Consumo } \\
\text { de P pastagem }\end{array}$} & \multicolumn{3}{|c|}{ P suplementar } & \multicolumn{3}{|c|}{ Balanço estimado de $\mathrm{P}^{(2)}$} \\
\hline & & & & & $\mathrm{MMC}$ & MM & $\overline{\mathrm{MMR}}$ & $\mathrm{MMC}$ & MM & MMR \\
\hline $1-3$ & Pico de lactação & $15 / 11-15 / 2$ & 16 a 17 & 12,6 & $\frac{\text { por dia) }}{7}$ & 7 & 7 & 2,6 & 2,6 & 2,6 \\
\hline $4-6$ & 1/3 inicial gestação & $15 / 2-15 / 4$ & 13 a 15 & 12,7 & 7 & 7 & 7 & 4,7 & 4,7 & 7,7 \\
\hline $7-9$ & $1 / 3$ médio da gestação & $15 / 4-15 / 8$ & 10 & 11,2 & 5,2 & 5,$2 ; 0$ & 5,$2 ; 15$ & 6,4 & 6,$4 ; 1,2$ & 6,$4 ; 16,2$ \\
\hline $9-12$ & 1/3 final da gestação & $15 / 8-15 / 10$ & 14 & 11,8 & 5,2 & 0 & 15 & 3 & 2,2 & 12,8 \\
\hline & & $15 / 10-15 / 11$ & 14 & 13,6 & 7 & 7 & 7 & 6,6 & 6,6 & 6,6 \\
\hline
\end{tabular}

${ }^{(1)}$ De acordo com o National Research Council (1996). ${ }^{(2)}$ Balanço estimado de $\mathrm{P}$ = consumo - demanda. MMC, mistura mineral completa; MM, MMC sem fosfato bicálcico; MMR, MM + concentrado. 
padrões norte-americanos (National Research Council, 2001), mas não pelos britânicos (Agricultural and Food Research Council, 1991). Ferris et al. (2010) ressaltam a importância da utilização de variáveis fisiológicas, menos sujeitas às interferências do manejo, nas avaliações de reprodução.

A retirada da fonte de $\mathrm{P}$ do suplemento mineral, durante a estação seca, não ocasionou prejuízo ao desempenho reprodutivo das vacas nos seis anos avaliados. As vacas do tratamento sem P suplementar, na estação seca, apresentaram desempenho reprodutivo (taxa de prenhez, intervalo de partos e o retorno da atividade cíclica ovariana) semelhante ao das vacas dos outros tratamentos (Tabela 5). A média geral do intervalo entre partos foi de 364,8 dias, considerado ideal, pois permite que as vacas produzam um bezerro por ano. Além disso, não se encontraram diferenças nas concentrações médias de progesterona entre as vacas, medidas desde 30 dias após o parto. Foram considerados os níveis plasmáticos de progesterona acima de $1 \mathrm{ng} \mathrm{mL} \mathrm{m}^{-1}$, que são indicativos de início de atividade cíclica ovariana em vacas em anestro, apesar de valores mais baixos terem sido relatados em vacas zebuínas (Ferreira et al., 2007). Com base nos níveis plasmáticos de progesterona e na confirmação de corpo lúteo cíclico, o retorno da atividade cíclica ovariana foi

Tabela 4. Concentrações médias plasmáticas de progesterona, ureia, glicose, albumina e fósforo inorgânico (Pi) de vacas Nelore paridas, que receberam diferentes suplementos na estação $\operatorname{seca}^{(1)}$.

\begin{tabular}{lccccc}
\hline Tratamento & $\begin{array}{c}\text { Progesterona } \\
\left(\mathrm{ng} \mathrm{dL}^{-1}\right)\end{array}$ & Ureia & Glicose & Albumina & Pi \\
\hline MMC & $2,38 \pm 3,11 \mathrm{a}$ & $10,94 \pm 6,42 \mathrm{a}$ & $93,16 \pm 19,79 \mathrm{a}$ & $3,89 \pm 0,49 \mathrm{a}$ & $4,91 \pm 0,26 \mathrm{~b}$ \\
MM & $1,95 \pm 2,33 \mathrm{a}$ & $12,19 \pm 7,56 \mathrm{a}$ & $85,98 \pm 16,42 \mathrm{a}$ & $4,02 \pm 0,51 \mathrm{a}$ & $4,96 \pm 0,18 \mathrm{~b}$ \\
MMR & $1,53 \pm 2,09 \mathrm{a}$ & $10,51 \pm 4,46 \mathrm{a}$ & $87,61 \pm 19,22 \mathrm{a}$ & $3,93 \pm 0,64 \mathrm{a}$ & $5,41 \pm 0,24 \mathrm{a}$ \\
\hline
\end{tabular}

${ }^{(1)}$ Médias \pm desvio-padrão nas linhas não diferem entre si, pelo teste $\mathrm{SNK}$, a $5 \%$ de probabilidade. MMC, mistura mineral completa; $\mathrm{MM}=\mathrm{MMC}$ sem fosfato bicálcico; $\mathrm{MMR}=\mathrm{MM}+$ concentrado. de 51,$17 ; 50,47$ e 50,94 dias, respectivamente para MMC, MM e MMR.

Considerando-se os valores limite dos indicadores metabólicos do status nutricional de gado de corte sugeridos - glicose inferior a $40 \mathrm{mg} \mathrm{dL}^{-1}$, albumina inferior a $3,0 \mathrm{~g} \mathrm{dL}^{-1}$, e ureia inferior a $15 \mathrm{mg} \mathrm{dL}$ (Diaz González et al., 2000) -, as concentrações de ureia das vacas no período puerperal (início da estação chuvosa) do quinto ano experimental estão baixas (Tabela 5). É comum a redução na concentração de ureia no sangue, no início da lactação, como neste caso, quando a ureia está com tendência de apresentar maior variação do que outro indicador do status de proteína - a albumina. A ureia indica o status proteico a curto prazo, enquanto a albumina indica o status a um prazo mais longo (Diaz González et al., 2000). Segundo Diaz González et al. (2000), a fertilidade da vaca tende a se reduzir, caso a concentração de albumina seja inferior a $3,0 \mathrm{mg} \mathrm{dL}^{-1}$. As concentrações médias de albumina, em todos os tratamentos, estiveram acima desse valor. Somado ao bom desempenho reprodutivo das vacas, a baixa ureia circulante pode apenas indicar um desequilíbrio momentâneo no balanço de proteína:energia no rúmen.

Vacas primíparas têm maior demanda de $\mathrm{P}$ na dieta. Odongo et al. (2007) estudaram o desempenho de vacas leiteiras, por duas lactações completas, e relataram queda de $11 \%$ na ingestão de MS na segunda lactação de vacas leiteiras primíparas, que ingeriram dieta com $0,35 \%$ de $\mathrm{P}$, em comparação a aquelas que receberam dieta com $0,42 \%$ de $\mathrm{P}$. Vacas na dieta marginal exibiram menor peso vivo, menor condição corporal e maior número de dias em que estavam "vazias" $(\mathrm{p}<0,10)$ do que aquelas com $0,42 \%$ de $\mathrm{P}$. O intervalo entre parições foi de 14,2 meses para as vacas com $0,35 \% \mathrm{P}$ e de 12,9 meses para aquelas com $0,42 \%$ P. Os autores concluíram que o fornecimento

Tabela 5. Taxas de prenhez (\%) e retorno da atividade cíclica ovariana pós parto (PS) e intervalo de partos (IEP), em vacas Nelore, nos tratamentos com suplementação diferenciada na estação seca ${ }^{(1)}$.

\begin{tabular}{|c|c|c|c|c|c|c|c|c|c|c|}
\hline \multirow[t]{2}{*}{ Tratamento } & \multicolumn{6}{|c|}{ Ano } & \multirow{2}{*}{$\begin{array}{c}\text { Média } \\
(\%)\end{array}$} & \multicolumn{3}{|c|}{ Retorno da atividade cíclica dias } \\
\hline & 1 & 2 & 3 & 4 & 5 & 6 & & $\mathrm{n}$ & PS & IEP \\
\hline MMC & $100(19 / 19)$ & $73,7(14 / 19)$ & $100(19 / 19)$ & $100(19 / 19)$ & $94,7(18 / 19)$ & $89,5(17 / 19)$ & $93,2 \mathrm{a}$ & 17 & $51,17 \pm 12,04 a$ & $362,5 \pm 2,36 a$ \\
\hline MM & $100(19 / 19)$ & $78,9(15 / 19)$ & $84,2(16 / 19)$ & $78,9(15 / 19)$ & $94,7(18 / 19)$ & $84,2(16 / 19)$ & $86,8 \mathrm{a}$ & 17 & $50,47 \pm 12,71 \mathrm{a}$ & $367,8 \pm 2,50 \mathrm{a}$ \\
\hline MMR & $94,4(17 / 18)$ & $77,8(14 / 18)$ & $94,4(17 / 18)$ & $88,9(16 / 18))$ & $100(18 / 18)$ & $94,4(17 / 18)$ & $91,7 \mathrm{a}$ & 18 & $50,94 \pm 14,11 \mathrm{a}$ & $364,7 \pm 2,46 a$ \\
\hline
\end{tabular}

${ }^{(1)}$ Médias seguidas de letras iguais, nas colunas, não diferem, a 5\% de probabilidade, pelo $\chi^{2}$ calculado $(0,694)$ para a taxa de prenhez, e pelo teste $\mathrm{F}$ para PS e IEP. MMC, mistura mineral completa; MM, MMC sem fosfato bicálcico; MMR, MM + concentrado. Valores entre parênteses referem-se ao número de vacas prenhez/total de vacas. 
de dieta mais rica em $\mathrm{P}$ pode ser importante para as vacas primíparas, pela combinação das demandas de produção de leite e crescimento. No presente trabalho, o arraçoamento na estação seca favoreceu a nova concepção de vacas primíparas, que apresentaram em média 12 e 19 dias a menos de intervalo de partos do que as vacas dos tratamentos MMC e MM, respectivamente. $\mathrm{O}$ contraste $\mathrm{MMC}, \mathrm{MM} \times \mathrm{MMR}$, e vacas primíparas $\mathrm{x}$ vacas multíparas foi significativo para essa variável $(\mathrm{p}=0,0161)$.

\section{Conclusão}

A retirada do fosfato bicálcico suplementar, fonte de fósforo e cálcio, na estação seca, não prejudica o desempenho de vacas multíparas em pastejo de capim-marandu.

\section{Agradecimentos}

À Fundação de Apoio ao Desenvolvimento do Ensino, Ciência e Tecnologia do Estado de Mato Grosso do Sul, pelo financiamento da pesquisa e concessão de bolsa; ao Conselho Nacional de Desenvolvimento Científico e Tecnológico, por concessão de bolsas; a Rafael Geraldo de Oliveira Alves, pelo auxílio no delineamento estatístico; a Eustáquio Camargo Vaz (in memorian), pelo manejo dos animais; a Gustavo Eugênio Barrocas, pelo auxílio no laboratório; às bolsistas Elizângela Luiz Caxias e Simone da Cunha Pereira Lopes e a Jacir Vieira de Barros e José Porfírio Ribeiro, pelo acompanhamento dos trabalhos em campo.

\section{Referências}

AGRICULTURAL AND FOOD RESEARCH COUNCIL. Technical Committee on Responses to Nutrients. Commonwealth Agricultural Bureaux International. A reappraisal of the calcium and phosphorus requirements of sheep and cattle. Nutrition Abstracts and Reviews. Series B: Livestock Feeds and Feeding, v.61, p.573-612, 1991.

ALVES, A.A.; SALLES, R. de O.; AZEVEDO, D.M.M.R.; AZEVEDO, A.R. de. Fatores que interferem no consumo de alimento pelo ruminantes: uma revisão. Revista Cientifica de Produção Animal, v.3, p.67-72, 2001.

DIAZ GONZÁLEZ, F.H.; BARCELLOS, J.O.; OSPINA PATIÑO, H.; RIBEIRO, L.A.O. Perfil metabólico em ruminantes: seu uso em nutrição e doenças nutricionais. Porto Alegre: UFRGS, 2000. 106p.

EKELUND, A.; SPÖRNDLY, R.; HOLTENIUS, K. Influence of low phosphorus intake during early lactation on apparent digestibility of phosphorus and bone metabolism in dairy cows. Livestock Science, v.99, p.227-236, 2006.

EUCLIDES, V.P.B.; EUCLIDES FILHO, K. Uso de animais na avaliação de forrageiras. Campo Grande: Embrapa-CNPGC, 1998. 59p. (Embrapa-CNPGC. Documentos, 74).

FERREIRA, M.B.D.; LOPES, B.C.; AZEVEDO, N.A.; FERNANDES, L.O; RIBEIRO, S.H.A.; MARQUES JUNIOR, C.A.; ROCHA, A.A. Descrição de ciclo estral curto no puerpério de multíparas Bos taurus indicus da raça Gir leiteiro. FAZU em Revista, n.4, p.68-72, 2007.

FERRIS, C. Reducing dietary phosphorus inputs within dairy systems. Hillsborough: AgriSearch, 2010. p.49-85. Available at: $<$ http://www.afbini.gov.uk/agrisearch-improve-dairy-farmingsustainability.pdf\#page=54>. Accessed on: 23 Nov. 2011.

FERRIS, C.P.; MCCOY, M.A.; PATTERSON, D.C.; KILPATRICK, D.J. Effect of offering dairy cows diets differing in phosphorus concentration over four successive lactations: 2. Health, fertility, bone phosphorus reserves and nutrient utilisation. Animal, v.4, p.560-571, 2010.

FICK, K.R.; MCDOWELL, L.R.; MILES, J.H.;WILKINSON, N.S.; FUNK, J.D.; CONRAD, J.H.; DAYRELL, M.de S.; ROSA, I.V. Métodos de análises de minerais em tecidos de animais e de plantas. 2.ed. Gainesville: University of Florida, 1980. Paginação irregular.

HARRIS, L.E. Os métodos químicos e biológicos empregados na análise de alimentos. Gainesville: University of Florida, 1970. Paginação irregular.

HAYDOCK, K.P.; SHAW, N.H. The comparative yield method for estimating dry matter yield of pasture. Australian Journal of Experimental Agriculture and Animal Husbandry, v.15, p.663-670, 1975.

KARN, J.F. Phosphorus nutrition of grazing cattle: a review. Animal Feed Science and Technology, v.89, p.133-153, 2001.

KNOWLTON, K.F.; RADCLIFFE, J.S.; NOVAK, C.L.; EMMERSON, D.A. Animal management to reduce phosphorus losses to the environment. Journal of Animal Science, v.82, p.E173-E195, 2004.

LOPEZ, H.; KANITZ, F.D.; MOREIRA, V.R.; WILTBANK, M.C.; SATTER, L.D. Effect of dietary phosphorus on performance of lactating dairy cows: milk production and cow health. Journal of Dairy Science, v.87, p.139-145, 2004a.

LOPEZ, H.; WU, Z.; SATTER, L.D.; WILTBANK, M.C. Effect of dietary phosphorus concentration on estrous behavior of lactating dairy cows. Theriogenology, v.61, p.437-445, $2004 \mathrm{~b}$.

MACHADO, R.; CORRÊA, R.F.; BARBOSA, R.T.; BERGAMASCHI, M.A.C.M. Escore da condição corporal e sua aplicação no manejo reprodutivo de ruminantes. São Carlos: Embrapa Pecuária Sudeste, 2008. (Embrapa Pecuária Sudeste. Circular técnica, 57).

MCIVOR, J.G.; GUPPY, C.; PROBERT, M.E. Phosphorus requirements of tropical grazing systems: the northern Australian experience. Plant and Soil, v.349, p.55-67, 2011.

MORAES, S. da S. Importância da suplementação mineral para bovinos de corte. Campo Grande: Embrapa Gado de Corte, 2001. 
26p. (Embrapa Gado de Corte. Documentos, 114). Disponível em: $<$ http://www.cnpgc.embrapa.br/publicacoes/doc/doc114/>. Acesso em: 25 nov. 2011.

NATIONAL RESEARCH COUNCIL. Nutrient requirements of beef cattle. $7^{\text {th }}$ ed.rev. Washington: NRC, 1996. 242p.

NATIONAL RESEARCH COUNCIL. Nutrient requirements of dairy cattle. $7^{\text {th }}$ ed.rev. Washington: NRC, 2001. 408p.

NICODEMO, M.L.F.; SERENO, J.R.B.; AMARAL, T.B. Minerais na eficiência reprodutiva de bovinos. São Carlos: Embrapa Pecuária Sudeste, 2008. 69p. (Embrapa Pecuária Sudeste. Documentos, 80).

ODONGO, N.E.; MCKNIGHT, D.; KOEKKOEK, A.; FISHER, J.W.; SHARPE, P.; KEBREAB, E.; FRANCE, J.; MCBRIDE, B.W. Long-term effects of feeding diets without mineral phosphorus supplementation on the performance and phosphorus excretion in high-yielding dairy cows. Canadian Journal of Animal Science, v.87, p.639-646, 2007.

PIMENTEL, J.J. de O.; LANA, R. de P.; GRAÇA, D. de S.; MATOS, L.L. de; TEIXEIRA, R.F.A. Teores de proteína bruta no concentrado e níveis de suplementação para vacas leiteiras em pastagens de capim-braquiária cv. Marandu no período da seca. Revista Brasileira de Zootecnia, v.40, p.418-425, 2011.
PUGGAARD, L.; KRISTENSEN, N.B.; SEHESTED, J. Effect of decreasing dietary phosphorus supply on net recycling of inorganic phosphate in lactating dairy cows. Journal of Dairy Science, v.94, p.1420-1429, 2011.

SANTOS, M.E.R.; FONSECA, D.M. da; EUCLIDES, V.P.B; RIBEIRO JÚNIOR, J.I.; NASCIMENTO JÚNIOR, D. do; MOREIRA, L. de M. Produção de bovinos em pastagens de capim-braquiária diferidas. Revista Brasileira de Zootecnia, v.38, p.635-642, 2009.

SATTER, L.D.; KLOPFENSTEIN, T.; ERICKSON, G.E.; POWELL, J.M. Phosphorus and dairy/beef nutrition. Madison: American Society of Agronomy, 2005. p.587-606. Available at: $<$ http://digitalcommons.unl.edu/cgi/viewcontent.cgi? article $=1564$ \&context=animalscifacpub $>$. Accessed on: 26 Nov. 2011.

TALLAM, S.K.; EALY, A.D.; BRYAN, K.A.; WU, Z. Ovarian activity and reproductive performance of dairy cows fed different amounts of phosphorus. Journal of Dairy Science, v.88, p.3609-3618, 2005.

WU, Z.; SATTER, L.D. Milk production and reproductive performance of dairy cows fed two concentrations of phosphorus for two years. Journal of Dairy Science, v.83, p.1052-1063, 2000 .

Recebido em 25 de março de 2011 e aprovado em 30 de novembro de 2011 